“( 2015 IEEE. Personal use of this material is permitted. Permission from IEEE must be obtained for all other uses, in any current or future media, including reprinting/republishing this material for advertising or promotional purposes, creating new collective works, for resale or redistribution to servers or lists, or reuse of any copyrighted component of this work in other works." 


\title{
Optimum Experimental Design applied to MEMS accelerometer calibration for 9-parameter auto-calibration model
}

\author{
Lin Ye, Steven W. Su
}

\begin{abstract}
Optimum Experimental Design (OED) is an information gathering technique used to estimate parameters, which aims to minimize the variance of parameter estimation and prediction. In this paper, we further investigate an OED for MEMS accelerometer calibration of the 9-parameter auto-calibration model. Based on a linearized 9-parameter accelerometer model, we show the proposed OED is both G-optimal and rotatable, which are the desired properties for the calibration of wearable sensors for which only simple calibration devices are available. The experimental design is carried out with a newly developed wearable health monitoring device and desired experimental results have been achieved.
\end{abstract}

\section{INTRODUCTION}

Owing to the rapid development of Micro-ElectroMechanical systems (MEMS), the performance of chip-based sensors improved rapidly in terms of size, weight, cost and power consumption. Today, MEMS accelerometers are widely-used in several application areas. Due to their size and weight advantages, these sensors have already been extensively utilized in wearable health monitoring devices [1] and consumer electronic devices, such as smartphone and smart watch. Because of the bias instability and output noise of MEMS sensors, calibration is necessary to obtain an accurate measurement for most applications mentioned above. However, most users, even some of the researchers are lacking sophisticated laboratory equipments (e.g., rotary table) to implement classical calibration [2] experiment.

Recently, several papers [3]-[5] developed a new calibration method for triaxial accelerometer, knowing as autocalibration, which can be implemented without sophisticated laboratory equipments. However, the calibration quality has not received the attention it deserves, particularly for each individual calibration. To authors' best knowledge, most studies focus on the algorithms of parameter estimation and their feasibility investigation. Few papers roughly described the selections of experimental observations. Paper [6] provided a systematic investigation of ODE for auto-calibration of tri-axial accelerometers.

This paper adopts the linearization method for the 9parameter auto-calibration model from [6]. After that, the 12observation design proposed in [6] is further investigated in order to remove inertial misalignment error (i.e., installation error). We prove the 12-observation experimental design [6] is both G-optimal and rotatable.

L. Ye and S. W. Su are with the Centre for Health Technology, Faculty of Engineering and Information Technology, University of Technology, Sydney, Australia Lin.Ye-1estudent.uts.edu.au, Steven.SuQuts.edu.au
To test the effectiveness of both the linearization method and the optimum experimental design, the ODE based caliberation has been realized in an recently developed wearable health monitoring device without using sophisticated laboratory equipments. Generally, most of the existing algorithms in literatures are too complicated to be implemented in microcontroller for online auto-calibration. In this study, the proposed auto-calibration procedure has been successfully implemented in the microcontroller of the wearable device to perform accurate calibration directly after experiments. Discussion of the experimental results has been provided.

\section{OPTIMUM EXPERIMENTAL DESIGN}

\section{A. Linearization of the 9-parameter auto-calibration model}

Extensive research has been done on auto-calibration of triaxial accelerometers. Generally, a better algorithm of parameter estimation can reduce the error. For further improvement, a set of well selected observation points can also improve calibration accuracy, which can be achieved by experimental design [7].

To apply optimum experimental design for auto-calibration efficiently, it is necessary to simplify the model. A 9parameter triaxial accelerometer can be expressed as

$$
\left[\begin{array}{l}
a_{x} \\
a_{y} \\
a_{z}
\end{array}\right]=\left[\begin{array}{ccc}
S_{x x} & S_{x y} & S_{x z} \\
S_{x y} & S_{y y} & S_{y z} \\
S_{x z} & S_{y z} & S_{z z}
\end{array}\right]\left(\left[\begin{array}{c}
v_{x} \\
v_{y} \\
v_{z}
\end{array}\right]+\left[\begin{array}{c}
o_{x} \\
o_{y} \\
o_{z}
\end{array}\right]\right),
$$

where vector $A=\left[\begin{array}{lll}a_{x}, & a_{y}, & a_{z}\end{array}\right]^{T}$ is the acceleration component, $V=\left[\begin{array}{ccc}v_{x}, & v_{y}, & v_{z}\end{array}\right]^{T}$ is the raw acceleration from sensor, and $O=\left[\begin{array}{ccc}o_{x} & o_{y} & o_{z}\end{array}\right]^{T}$ is offset. The diagonal elements of scale factor matrix $\left(S_{i i}\right)$ are sensitivity factors of each direction while off-diagonal elements $\left(S_{i j}\right)$ are sensitivity factors of cross-axis sensitivity [3].

The method of auto-calibration is based on the fact that the overall acceleration of each axis is equal to the magnitude of local gravity acceleration. To be specific, the magnitude is " $1 \mathrm{~g}$ " in static state condition,

$$
g=\sqrt{a_{x}^{2}+a_{y}^{2}+a_{z}^{2}} .
$$

By applying the auto-calibration method for 9-parameter triaxial accelerometer model in Eq.(1), we have

$$
g^{2}=\sum_{i=x, y, z}\left[\sum_{j=x, y, z} S_{i j} \cdot\left(V_{j}+O_{j}\right)\right]^{2}+\varepsilon_{r},
$$

where $S_{i j}=S_{j i}, \varepsilon_{r}$ is the squared difference between raw acceleration and local gravity [3]. 
It is infeasible to propose an experimental design for Eq.(3), with all unknown parameters and their intersections. For this reason, to apply optimum experimental design, a practical linearization method is necessary to simplify Eq.(3) into a second-order polynomial form [8],

$$
y=\beta_{0}+\sum_{1}^{m} \beta_{j} x_{j}+\sum_{j=1}^{m-1} \sum_{k=j+1}^{m} \beta_{j k} x_{j} x_{k}+\sum_{j=1}^{m} \beta_{j j} x_{j}^{2} .
$$

To approach this simplification, firstly, terms with inappreciable effect should be neglected. According to datasheets, for most newly developed IMU products, the off-diagonal sensitivity factors $\left(S_{i j}\right)$ are less than $1 \%$ of diagonal sensitivity factors $\left(S_{i i}\right)$. The maximum zero-g initial offsets of $\mathrm{x}$ and $\mathrm{y}$ axis are normally less than $\pm 0.1 \mathrm{~g}$ while the offset for $\mathrm{z}$ axis is less than $\pm 0.15 \mathrm{~g}$. As worst initial offset is quite large, a pre-calibration is recommended. The pre-calibration can reduce zero-g initial offset and determine the polarity of scale factors. After this procedure, the off-diagonal sensitivity factors and the residual offsets in Eq.(3) are small enough to be neglected, particularly for their products. We remove all terms contained $S_{i j} O_{i}, S_{i j} S_{j k}, O_{i}^{2}$ and $O_{i} O_{j}$ [6]. The remaining equation is

$$
\begin{aligned}
g^{2}= & \sum_{i=x, y, z} S_{i i}^{2} V_{i}^{2}+\sum_{i=x, y, z} 2 S_{i i}^{2} V_{i} O_{i} \\
& +\sum_{i \neq j}\left(S_{i i}+S_{j j}\right) S_{i j} V_{i} V_{j}+\tilde{\varepsilon}+\bar{\varepsilon}
\end{aligned}
$$

where $S_{i j}=S_{j i}$ for 9-parameter model, $\tilde{\varepsilon}$ is zero mean noise and $\bar{\varepsilon}$ is non-zero error representing the means of the summation of the neglected terms.

Eq.(1) shows that our original model contains 9 unknown parameters. The desired simplification is that Eq.(5) can be described by the form of Eq.(4) with 9 unknown parameters too. To achieve this goal, we apply parameter re-combined technique to define new 9 known parameters as follows [6]:

$$
\begin{array}{cc}
S_{x x}^{2}=\beta_{11} & 2 S_{x x}^{2} O_{x}=\beta_{1} \\
S_{y y}^{2}=\beta_{22} & 2 S_{y y}^{2} O_{y}=\beta_{2} \\
S_{z z}^{2}=\beta_{33} & 2 S_{z z}^{2} O_{z}=\beta_{3} \\
2\left(S_{x x}+S_{y y}\right) S_{x y}=\beta_{12} \\
2\left(S_{x x}+S_{z z}\right) S_{x z}=\beta_{13} \\
2\left(S_{y y}+S_{z z}\right) S_{y z}=\beta_{23}
\end{array}
$$

Replacing $V_{i, j, k}$ with $x_{1,2,3}$, Eq.(5) without neglected terms can be expressed as:

$$
y=\sum_{j=1}^{3} \beta_{j} x_{j}+\sum_{j=1}^{2} \sum_{k=j+1}^{3} \beta_{j k} x_{j} x_{k}+\sum_{j=1}^{3} \beta_{j j} x_{j}^{2}+\tilde{\varepsilon},
$$

which is a 9-unknown-parameter second-order polynomial model without interception.

\section{B. Experimental plan and its properties}

For the second-order polynomial model, Eq.(7), optimum experimental design has been applied to obtain a specific 12observation G-optimal design[6], Icosahedron design. Table I shows the Icosahedron design with $a=\sqrt{\frac{2}{5+\sqrt{5}}}$ and $b=$
TABLE I

TABLE I. THREE FACTORS ICOSAHEDRON DESIGN FOR TRI-AXIAL ACCELEROMETER MODEL

\begin{tabular}{|c||c|c|c|}
\hline Observation & $x_{1}$ & $x_{2}$ & $x_{3}$ \\
\hline \hline 1 & 0 & $\mathrm{a}$ & $\mathrm{b}$ \\
\hline 2 & 0 & $-\mathrm{a}$ & $\mathrm{b}$ \\
\hline 3 & 0 & $\mathrm{a}$ & $-\mathrm{b}$ \\
\hline 4 & 0 & $-\mathrm{a}$ & $-\mathrm{b}$ \\
\hline 5 & $\mathrm{a}$ & $\mathrm{b}$ & 0 \\
\hline 6 & $-\mathrm{a}$ & $\mathrm{b}$ & 0 \\
\hline 7 & $\mathrm{a}$ & $-\mathrm{b}$ & 0 \\
\hline 8 & $-\mathrm{a}$ & $-\mathrm{b}$ & 0 \\
\hline 9 & $\mathrm{~b}$ & 0 & $\mathrm{a}$ \\
\hline 10 & $-\mathrm{b}$ & 0 & $\mathrm{a}$ \\
\hline 11 & $\mathrm{~b}$ & 0 & $-\mathrm{a}$ \\
\hline 12 & $-\mathrm{b}$ & 0 & $-\mathrm{a}$ \\
\hline
\end{tabular}

$\frac{1+\sqrt{5}}{\sqrt{10+2 \sqrt{5}}}$. These 12 observations can form an Icosahedron in three dimension rectangular coordinate system.

According to [6], in this study, we found that the Icosahedron design is rotatable. From the definition of rotatability, a design is rotatable if the variance of the predicted response only depends on the distance between observation points from center point [7].

In order to analysis the relation between G-optimal and rotatable for this experimental design, we use standardized variance $d=(x, \xi)$ instead of variance $\operatorname{var}(\hat{y}(x))$. Over the experimental design region $\chi, d=(x, \xi)$ is defined as:

$$
d(x, \xi)=f^{T}(x) M^{-1}(\xi) f(x)=\frac{N \operatorname{var}(\hat{y}(x))}{\sigma^{2}},
$$

where $\mathbf{N}$ is the number of observations [8] [9], and $f^{T}(x)$ is

$f^{T}(x)=\left[\begin{array}{lllllllll}x_{1} & x_{2} & x_{3} & x_{1}^{2} & x_{2}^{2} & x_{3}^{2} & x_{1} x_{2} & x_{1} x_{3} & x_{2} x_{3}\end{array}\right]$.

For an experimental design, rotatability is aiming to optimize the variance of every single observation during parameter estimation.

To discuss the rotatability of Icosahedron design in Table I, let us recall Eq.(7) for which the prediction variance $\hat{y}(x)$ is

$$
\operatorname{var}(\hat{y}(x))=\sigma^{2} f^{T}(x)\left(X^{T} X\right)^{-1} f(x) .
$$

The measurement matrix $X \in R^{12 \times 9}$ in (10) can be easily derived from Table I,

$\mathrm{X}=\left[\begin{array}{ccccccccc}x_{1} & x_{2} & x_{3} & x_{1}^{2} & x_{2}^{2} & x_{3}^{2} & x_{1} x_{2} & x_{1} x_{3} & x_{2} x_{3} \\ 0 & -a & -b & 0 & a^{2} & b^{2} & 0 & \ldots & \ldots \\ 0 & -a & b & 0 & a^{2} & b^{2} & 0 & \ldots & \ldots \\ \vdots & \vdots & \vdots & \vdots & \vdots & \vdots & \vdots & \ddots & \\ \vdots & \vdots & \vdots & \vdots & \vdots & \vdots & \vdots & & \ddots\end{array}\right]$.

Overall, we have

$$
\begin{aligned}
d(x, \xi) & =\frac{N \operatorname{var}(\hat{y}(x))}{\sigma^{2}} \\
& =3\left(x_{1}^{2}+x_{2}^{2}+x_{3}^{2}\right)+6\left(x_{1}^{2}+x_{2}^{2}+x_{3}^{2}\right)^{2} .
\end{aligned}
$$


From Eq.(12), the standardized variance is only related to $x_{1}^{2}+x_{2}^{2}+x_{3}^{2}$, which indicates that the variance only depends on the distance of observation points and center point, hence Icosahedron design is rotatable [10] [11].

Let us recall the definition of G-optimal for experimental design, if

$$
\max _{x \in R}\{d(x, \xi)\}=p,
$$

where $p$ is the number of unknown parameters, the design is G-optimal [8]. For getting the maximum value of $d(x, \xi)$, the overall acceleration in static state is equal to magnitude of local gravity " $1 \mathrm{~g}$ " due to the constraint $\left(x_{1}^{2}+x_{2}^{2}+x_{3}^{2}=1\right)$. In this case, from (12), we have

$$
\max _{x \in R}\{d(x, \xi)\}=3+6=9 .
$$

It proves that Icosahedron experimental design is also Goptimal.

The Icosahedron design is not only G-optimal but also rotatable which indicates the estimation of unknown parameters has the minimum maximum variance and all observations share the same prediction variance for model (7).

The practical application of the rotatability in calibration is reducing the misalignment error. Generally, it is infeasible to evaluate the misalignment error when implementing an experimental design. Assuming the tilt angles between MEMS accelerometer and device are $\theta_{x}, \theta_{y}$ and $\theta_{z}$, then three rotation matrices can be defined as:

$$
\begin{aligned}
& R x=\left[\begin{array}{ccc}
1 & 0 & 0 \\
0 & \cos \theta_{x} & -\sin \theta_{x} \\
0 & \sin \theta_{x} & \cos \theta_{x}
\end{array}\right], \\
& R y=\left[\begin{array}{ccc}
\cos \theta_{y} & 0 & \sin \theta_{y} \\
0 & 1 & 0 \\
-\sin \theta_{y} & 0 & \cos \theta_{y}
\end{array}\right], \\
& R z=\left[\begin{array}{ccc}
\cos \theta_{z} & -\sin \theta_{z} & 0 \\
\sin \theta_{z} & \cos \theta_{z} & 0 \\
0 & 0 & 1
\end{array}\right] .
\end{aligned}
$$

Now, let us define a matrix which is the experiment in Table I, $D=\left[x_{1}, x_{2}, x_{3}\right], D_{\text {new }}$ is the matrix $D$ after rotation,

$$
D_{n e w}=\left(R x \times R y \times R z \times D^{T}\right)^{T}=\left[\begin{array}{lll}
k_{1}, & k_{2}, & k_{3}
\end{array}\right] .
$$

Based on $D_{\text {new }}$, new $\mathrm{X}$ matrix $X_{r} \in R^{12 \times 9}$ is

$X_{r}=\left[\begin{array}{lllllllll}k_{1} & k_{2} & k_{3} & k_{1}^{2} & k_{2}^{2} & k_{3}^{2} & k_{1} k_{2} & k_{1} k_{3} & k_{2} k_{3}\end{array}\right]$.

It can be computed that the information matrix $X_{r}^{T} X_{r}$ is invariant when the tilt angles $\theta_{x}, \theta_{y}$ and $\theta_{z}$ are changing. More precisely, this experiment can remain G-optimal with any initial observation as long as all the 12 observations can be observed on the proposed points "relatively",

$$
\begin{aligned}
d\left(x, \xi_{r}\right) & =\frac{N \operatorname{var}(\hat{y}(x))}{\sigma^{2}} \\
& =N f^{T}(x)\left(X_{r}^{T} X_{r}\right)^{-1} f(x)=9 .
\end{aligned}
$$

\section{IMPLEMENTATION AND ANALYSIS}

To implement the proposed linearization method and experimental plan, we developed a health monitoring device contains SCM (TI F430), IMU (InvenSense MPU9150) in Centre for Health Technology, University of Technology,Sydney and featured with Bluetooth module for wireless communication. We also designed a platform by SolidWorks and printed it with $3 \mathrm{D}$ printer.

\section{A. Implementation of experimental design}

The expected value in table I indicates acceleration component on each axis,

$$
g_{x, y, z}^{\prime}=\cos \theta_{x, y, z}
$$

where $g^{\prime}\left(x_{1}, x_{2}, x_{3}\right)$ is the acceleration component on each axis and $\theta$ is the included angle between axis and local gravity. Figure 2 shows the arrangements of our device for 12 observations.
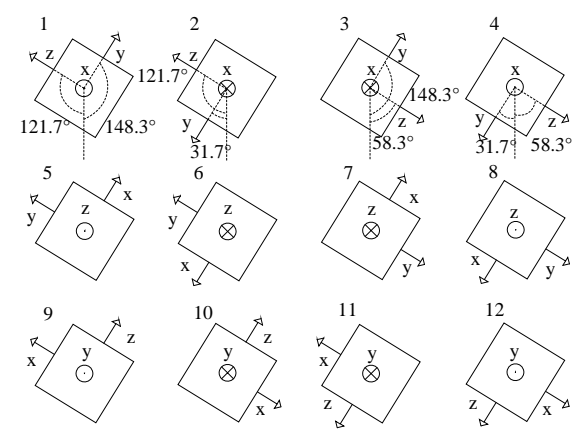

Fig. 1. The arrangements of MEMS accelerometer for Icosahedron design

In this experiment, we place our device on the surface of platform which is tilted $31.7^{\circ}$ from horizontal plane. The device is placed following the instrument in Fig.(1) that $x$, $\mathrm{y}$ and $\mathrm{z}$ indicate the positive direction of each axis. With the program we developed for this device, there will be 30 s to place the IMU in the correct direction before the device starts to record the data after we press the record button. When 12 observations complete, the device can calculate the parameters directly and store them for further data acquisition. This procedure can also be easily repeated to remove daily drift for accelerometer.

\section{B. Analysis of experimental data}

With the raw data of acceleration from 12 observations and auto-calibration model (3), weighted least square estimation (WLS) is able to estimate unknown parameters effectively if the data processing ability of SCM is considered,

$$
Y=X \beta+\bar{\varepsilon}+\tilde{\varepsilon} .
$$

In section 2, we explained that $\bar{\varepsilon}$ has inappreciable effect which can be neglected. Without $\bar{\varepsilon}$, we can apply WLS for Eq.(20) to solve unknown parameter $\hat{\beta}$ which is the recombination of $S$ and $O$. The result of parameter $\hat{\beta}$ is

$$
\hat{\beta}=\left(X^{T} W X\right)^{-1} X^{T} W Y,
$$


where the initial $\mathrm{X}$ is the raw acceleration data from 12 observations, $\mathrm{Y}$ is local gravity $1 \mathrm{~g}$, and $\mathrm{W}$ is weight matrix. In our model, weight matrix can be determined by the variance from several sets of experimental observations. Furthermore, some accelerometers have different sensitivity factors between $\mathrm{x}$, $\mathrm{y}$ axis and $\mathrm{z}$ axis. In this case, 8 observations contain the sensitivity factors of $\mathrm{z}$-axis should weight different with the other 4 observations. To simplify the calculation, we weight 12 observations equally. In order to estimate the unknown parameters precisely, a recursive analysis is necessary. The main idea of this recursive analysis is reducing non zero error $\bar{\varepsilon}$ to zero. Fig.(2) shows the procedure of the recursive analysis.

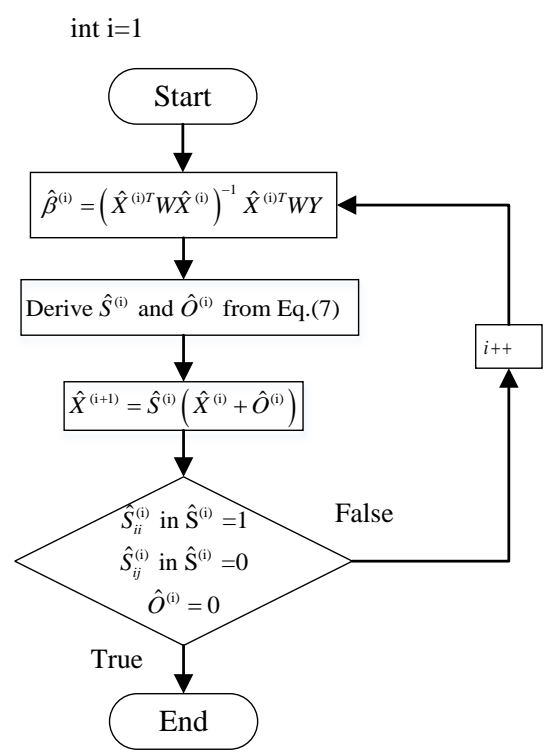

Fig. 2. The procedure of obtaining precise acceleration

To understand the procedure in Fig.(2), recall Eq.(1), $\hat{X}^{(i)}$ is representing measured acceleration components $V$ on each axis $\left(X^{(1)}\right.$ is the raw data). During the recursive analysis, $\hat{X}^{(i+1)}$ is closing to actual acceleration $A$ when comparing to $\hat{X}^{(i)}$. This will reduce the offset $\hat{O}^{(i)}$ and off-diagonal sensitivity factors $\hat{S}_{i j}^{(i)}$ which leads to the decrease of $\bar{\varepsilon}$ (each term in $\bar{\varepsilon}$ contains $\hat{O}^{(i)}$ and $\hat{S}_{i j}^{(i)}$ ). It means we can obtain better results by applying WLS due to the decrease of neglected terms $\bar{\varepsilon}[6]$.

When the offset $\hat{O}^{(i)}$ and off-diagonal sensitivity factors $\hat{S}_{i j}^{(i)}$ is zero(almost zero for practical situation), the final acceleration can then be expressed as:

$\hat{A}^{(n)}=\hat{S}^{(n)}\left(\cdots \hat{S}^{(2)}\left(\hat{S}^{(1)}\left(X^{(1)}+\hat{O}^{(1)}\right)+\hat{O}^{(2)}\right) \cdots+\hat{O}^{(n)}\right)$.

To verify the performance of this experimental design and parameter estimation on our device, we acquired another 25 observations randomly. Figure 4 shows the results of observation before and after calibration.

As we can see from Fig.(3), the magnitude of overall acceleration after calibration is stable and closed to local

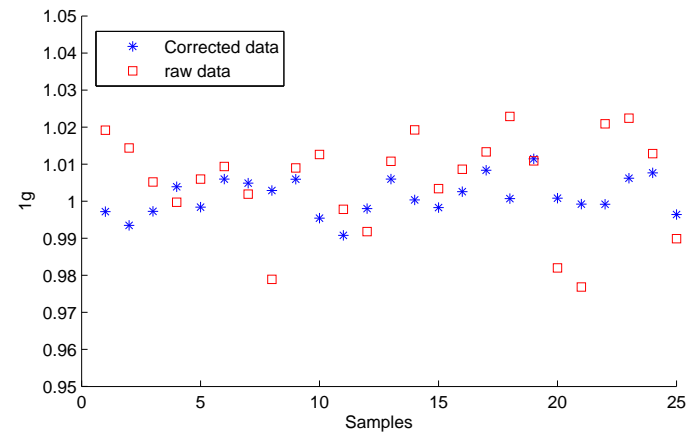

Fig. 3. Calibration results of 9-parameter accelerometer model

gravity $1 \mathrm{~g}$. The mean squared error (MSE) before calibration is $1.98 \times 10^{-4} g^{2}$ which reduces to $2.58 \times 10^{-5} g^{2}$ after we repeat the recursive algorithm for 3 times.

\section{CONCLUSIONS}

This paper further explores the optimal experimental design of the auto-calibration approach proposed in [6] for 9 parameter model of MEMS triaxial accelerometers. The optimal experimental design has been implemented in a recently developed IMU based wearable health monitoring device. Consequently, the measurement error of the device has been greatly reduced by the developed auto-calibration procedure. Experimental results well demonstrate the efficiency of the proposed optimal experimental design. We hope the developed optimal experimental design approach and its associated parameter estimation algorithm can provide a practical way to improve the accuracy of the wearable IMU devices and at the same time to enhance the acceptance of wearable monitoring devices from medical professionals.

\section{REFERENCES}

[1] M. Yuwono, B. D. Moulton, S. W. Su, B. G. Celler, H. T. Nguyen, "Unsupervised machine-learning method for improving the performance of ambulatory fall-detection systems," Biomed Eng Online, vol 11(9), 2012.

[2] M. Pedley, "High Precision Calibration of a Three-Axis Accelerometer," Freescale Semiconductor Application Note, AN4399, Rev 1.

[3] I. Frosio, S. Stuani, N. A. Borghese, "Autocalibration of MEMS Accelerometer,' IEEE Trans. Instrum. Meas, vol. 58(6), pp. 20342041, 2009.

[4] M. Glueck, D. Oshinubi, P. Schopp, Y. Manoli, "Real-Time Autocalibration of MEMS Accelerometers," IEEE Trans. Instrum. Meas, vol 63(1), pp. 96-105, 2014.

[5] S. P. Won, F. Golnaraghi, "A Triaxial Accelerometer Calibration Method Using a Mathmatical Model," IEEE Trans. Instrum. Meas., vol 59(8), pp. 2144-2513, 2010.

[6] L. Ye, S. W. Su, "Experimental Design and Its Posterior Efficiency for the Calibration of Wearable Sensors," Journal of Intelligent Learning Systems and Applications, pp. 11-20, 2015.

[7] D. C. Montgomery, "Design and Analysis of Experiments," John Wiley and Son, 2005.

[8] A. Atkinson, A. Donev, R. Tobias. "Optimum Experimental Designs, with SAS," Oxford university press, 2007.

[9] A. I. Khuri, S. Mukhopadhyay, "Response surface methodology," Rev. Comput. Stat, vol 2(2), pp. 128-149, 2010.

[10] R. Myers, D. C. Montgomery, "Response Surface Methodology:Process and Product Optimization Using Designed Experiments," John Wiley and Son, 1995.

[11] D. Cook, V. Fedorov, "Constrained Optimization of Experimental Design," Statistics, vol. 26, pp. 129-148, 1995. 